\title{
Adaptación de la escala de job crafting: evidencias de validez en el contexto laboral argentino
}

\author{
Solana Salessi ${ }^{1}$
}

\begin{abstract}
RESUMEN
El término job crafting designa una forma específica de comportamiento proactivo por la cual el empleado realiza voluntariamente cambios en las demandas y/o recursos laborales para impactar positivamente en su propio trabajo. El objetivo de este estudio fue adaptar y validar la Escala de Job Crafting desarrollada por Tims, Bakker y Derks en una muestra de trabajadores argentinos. Se empleó un diseño empírico-instrumental transversal bi-etápico En la primera etapa se ejecutó un estudio sobre una muestra no probabilística de 229 (56\% varones; $M_{\text {edad }}=33$ años; $D E_{\text {edad }}=6.24$ años; $M_{\text {antigüedad }}=7$ años, $D E_{\text {antiguedad }}=3.81$ años) trabajadores de PYMES radicadas en la ciudad de Rosario. E1 análisis paralelo optimizado sugirió 4 factores con autovalores propios sobre el percentil 95, los que explicaban el $60 \%$ de la varianza del constructo. En la segunda etapa se estudió una nueva muestra no probabilística integrada por 449 trabajadores $167 \%$ mujeres; $M_{\text {edad }}=34.5$ años; $D E_{\text {edad }}=12.9 ; M_{\text {antigüedad }}=8.6$ años, $D E_{\text {antiguedad }}=10.21$ años) de diversas organizaciones localizadas en el centro-sur de Santa Fe. Análisis factoriales confirmatorios indicaron la adecuación de un modelo tetra-factorial con 19 items como variables observables. La versión adaptada de la escala mostró adecuada consistencia interna, confiabilidad compuesta y validez convergente-discriminante. Las correlaciones positivas significativas encontradas entre las puntuaciones en las escalas de job crafting, capital psicológico, implicación en el trabajo y personalidad proactiva indicaron validez concurrente. Los resultados obtenidos proporcionan evidencias de validez de la adaptación de la escala de job crafting en el contexto laboral argentino. Se discuten los hallazgos. Se señalan fortalezas, limitaciones e implicancias de la investigación realizada.
\end{abstract}

Palabras clave: comportamiento organizacional, instrumento de medida, validez estadística, job crafting.

\section{Adaptation of Job Crafting Scale: Evidence of validity in the Argentine Labor Context}

\begin{abstract}
The word job crafting designates a specific form of proactive behaviour whereby the employee voluntarily makes changes in job demands and resources to positively impact on their own job. The aim of this study was to assess the underlying structure of the Job Crafting Scale developed for Tims, Bakker y Derks (2012) in a sample of Argentinean workers. A cross-sectional, empirical-instrumental and bi-phase design was used. In the first stage a study was carried out on a non-probabilistic sample of $229 \quad(56 \%$ males, $M_{\text {age }}=33$ years, $D E_{\text {age }}=6.24$ years, $M_{\text {seniority }}=7$ years, $D E_{\text {seniority }}=3.81$ years) PYMES's workers of Rosario 's city. The optimized parallel analysis suggested 4 factors with eigenvalues of the $95^{\text {th }}$ percentile; which explained $60 \%$ of the variance of the construct. In the second stage a new non-probabilistic sample composed of 449 workers
\end{abstract}

1 Consejo Nacional de Investigaciones Científicas y Técnicas (CONICET), Centro de Investigación y Transferencia, Universidad Nacional de Rafaela, Pontificia Universidad Católica Argentina, Argentina; solanasalessi@unraf.edu.ar; https://orcid.org/0000-0001-9496-9493. 
(67\% women, $M_{\text {age }}=34.5$ years, $D E_{\text {age }}=12.9, M_{\text {seniority }}=8.6$ years, $D E_{\text {seniority }}=10.21$ years) of various organizations located in the center-south of Santa $\mathrm{Fe}$ was studied. Confirmatory factor analyses indicated the adequacy of a tetra-factorial model with 19 items as observable variables. The adapted version of the scale showed adequate internal consistency, composite reliability and convergent-discriminant validity. The significant positive correlations found between the scores in the scales of job crafting, psychological capital, job involvement and proactive personality indicated concurrent validity. The results obtained provide evidence of the validity of the adaptation of the job crafting scale in the Argentine labour context. The findings are discussed. Strengths, limitations and implications of the research carried out are indicated.

Keywords: organizational behaviour, measurement instrument, statistical validity, job crafting.

Hacia finales del siglo $\mathrm{XX}$, los estudios sobre comportamiento organizacional cambiaron radicalmente sus concepciones sobre motivación y desempeño. En contraposición a las perspectivas tradicionales inspiradas en los principios del conductismo, la psicología organizacional contemporánea otorga un rol protagónico al trabajador, considerándolo capaz de rediseñar su trabajo (Demerouti, 2014). La expresión job crafting, del inglés job (trabajo) y to craft (elaborar), fue introducida en el ámbito académico para dar cuenta de los cambios que los empleados realizan en su trabajo para alinearlo a sus preferencias, competencias y motivaciones (Demerouti \& Bakker, 2014; Tims, Derks, \& Bakker, 2016). Si bien la traducción más aproximada al español sería "elaboración del trabajo", esta expresión no ha logrado posicionarse en la literatura española y latinoamericana, conservándose el término inglés original.

Inicialmente, el job crafting fue definido como los cambios físicos y cognitivos que las personas realizan en sus tareas, o en los limites funcionales y relacionales de sus puestos, con el fin de adaptarlo a sus necesidades, habilidades y aspiraciones (Wrzesniewski \& Dutton, 2001). Los cambios físicos atañen a las alteraciones en la forma, el alcance o la cantidad de tareas. Vale decir, los empleados cambian sus responsabilidades laborales agregando tareas más allá de las definidas en la descripción de su puesto, suprimiendo tareas secundarias o irrelevantes, o modificando el tiempo y el esfuerzo invertido en la ejecución de su trabajo. Los cambios cognitivos, en tanto, se refieren al modo en cómo se percibe y resignifica el trabajo. Así, por ejemplo, un empleado puede modificar las percepciones sobre su función vislumbrando su puesto como parte de un engranaje más amplio, subrayando la importancia que tiene su trabajo para otras áreas, e identificando su contribución para la concreción de las metas y objetivos de la organización. Finalmente, las modificaciones en los limites relacionales y funcionales del puesto atañen a las interacciones que se establecen con otras posiciones y áreas, independientemente de las que formalmente corresponden al puesto. Es decir, al establecimiento de relaciones entre empleados de distinto nivel en el organigrama organizacional. Tal es el caso, por ejemplo, de un directivo o ejecutivo que establece relaciones de tutoría (del inglés mentoring) con los trabajadores más novatos de la compañia (Berg, Dutton, \& Wrzesniewski, 2013; Wrzesniewski \& Dutton, 2001).

Más recientemente, el constructo ha sido definido en los términos de un comportamiento discrecional, orientado a modificar las demandas y recursos 
laborales en busca de un equilibrio óptimo (Demerouti \& Bakker, 2014; Tims, Bakker, \& Derks, 2012, 2013). En otras palabras, un tipo específico de comportamiento proactivo de ajuste persona-ambiente (Salessi \& Omar, 2017), desde el momento en que comprende aquellas acciones tendientes a lograr mayor compatibilidad entre las habilidades e intereses del trabajador y las características de su puesto.

Las raíces del job crafting pueden rastrearse en las teorias de rediseño del trabajo (Demerouti, 2014; Tims \& Bakker, 2010). En términos generales, el diseño ha sido analizado desde dos perspectivas, usualmente identificadas como rediseño de arriba hacia abajo (del inglés top-down) y rediseño de abajo hacia arriba (del inglés bottom-up). Para el primer enfoque, es la administración organizacional la que define qué modificaciones son necesarias en la estructura o contenido del puesto. Para la segunda aproximación, en tanto, es el propio trabajador quien modifica ciertos aspectos de su puesto y del ambiente social de trabajo, buscando optimizar su rendimiento y lograr mayores niveles de bienestar (Demerouti \& Bakker, 2014). El job crafting se inscribe, precisamente, en esta segunda perspectiva desde el momento en que representa aquellos cambios que acontecen a nivel individual y son iniciados por el propio trabajador (Demerouti, 2014).

Si bien la investigación preliminar sobre la temática ha sido desarrollada desde la perspectiva del construccionismo social (Berg et al., 2013; Wrzesniewski \& Dutton, 2001), Tims y Bakker (2010) han propuesto una aproximación alternativa: el modelo de demandas y recursos laborales (DRL, Bakker \& Demerouti, 2017). Desde esta perspectiva, el job crafting consiste en una serie de modificaciones ejecutadas sobre las demandas y/o recursos laborales (Tims et al., 2012; Tims et al., 2013). Las demandas representan aquellos aspectos físicos, psicológicos, sociales u organizacionales del trabajo que suponen la inversión de esfuerzos sostenidos por parte del trabajador, con los consecuentes costos asociados para su salud y bienestar. Los recursos, en tanto, hacen referencia a todos aquellos aspectos laborales (físicos, psicológicos, sociales u organizacionales) que, o bien ayudan a lidiar con las demandas y amortiguar su potencial dañino, o bien estimulan el desarrollo del trabajador, potenciando su motivación y promoviendo su bienestar (Demerouti, 2014; Demerouti \& Bakker, 2014; Petrou, Demerouti, Peeters, Schaufeli, \& Hetland, 2012).

Desde el modelo DRL (Tims \& Bakker, 2010; Tims et al., 2012; Tims et al., 2013), entonces, el trabajador puede "reelaborar" su trabajo de tres maneras. Por un lado, disminuyendo las presiones obstaculizantes. Por ejemplo, organizando las tareas de modo tal de reducir la carga mental, o disminuyendo la frecuencia de contacto con compañeros de trabajo conflictivos. Por otro lado, aumentando sus recursos estructurales y/o sociales. En este sentido, por ejemplo, puede capacitarse en nuevas competencias, conocimientos y/o métodos de trabajo, solicitar retroalimentación a su supervisor para monitorear su desempeño, o pedir la colaboración de sus pares frente a los inconvenientes y dificultades en el desarrollo de su tarea. Finalmente, incrementan sus retos laborales. Por ejemplo, liderando la implementación de innovaciones en su área, participando de nuevos proyectos de la organización, asumiendo tareas adicionales, etc.

Desde los primeros estudios teóricos a comienzos del nuevo milenio, la investigación empírica sobre job crafting ha protagonizado un crecimiento 
exponencial y sostenido. Identificar la influencia de factores situacionales y disposicionales sobre este comportamiento, asi como descubrir su potencial efecto sobre otras variables y resultados organizacionalmente relevantes, han sido objetivos prioritarios de la investigación en la temática. En este sentido, entre los principales antecedentes del job crafting la investigación ha señalado tanto a factores contextuales como a diferencias individuales. Entre los primeros, ciertas características del puesto (tales como la autonomía y la carga de trabajo), relaciones positivas entre líderes y subordinados, y un ambiente de cambio organizacional han revelado ser predictores significativos de este comportamiento (van Dam, Nikolova, \& van Ruysseveldt, 2013; Walk \& Handy, 2018). Entre las variables disposicionales, los cinco grandes factores de personalidad han sido señalados (Bell \& Njoli, 2016) como antecedentes importantes del job crafting, desde el momento en que la extraversión y la agradabilidad promoverian el incremento de los recursos sociales, la apertura a la experiencia incentivaría la búsqueda de desafios, y la estabilidad emocional y responsabilidad facilitarian el manejo de presiones obstaculizantes y el desarrollo de recursos estructurales.

No obstante, la personalidad proactiva (Bateman \& Crant, 1993) parece constituir el antecedente más relevante de este tipo de comportamiento (Bakker, Ficapal-Cusí, Torrent-Sellens, Boada-Grau, \& Hontangas-Beltrán, 2018; Zhang, Lu, \& Li, 2018). En efecto, el metaanálisis de Rudolph, Katz, Lavigne y Zacher, (2017) efectuado sobre 122 muestras independientes (equivalentes a un total de 35650 trabajadores) subraya las fuertes vinculaciones entre ambas variables $\left(r_{c}=.54\right)$. Asimismo, ciertos recursos psicológicos, tales como la autoeficacia, el optimismo y la resiliencia también han sido reiteradamente sindicados por la literatura como predictores significativos del job crafting (Berdicchia, Nicolli \& Masino, 2016; CarvalhoChinelato, Ferreira, \& Valentini, 2015; Cenciotti et al., 2016; Tims, Bakker, \& Derks, 2014).

En cuanto a las consecuencias, su impacto favorable sobre el rendimiento y sobre diversas actitudes de los empleados se encuentra respaldado por múltiples evidencias. Así, por ejemplo, diversos estudios (Petrou et al., 2015; Tims, Bakker, \& Derks, 2015; Tims et al., 2013; Tims et al., 2016) destacan que los empleados que se involucran en el rediseño de sus puestos muestran mejoras significativas en su desempeño laboral y en sus niveles de bienestar psicológico, así como menor estrés e intenciones de desvincularse de la organización. Paralelamente, el job crafting demuestra estar asociado a actitudes positivas y estados de realización en el trabajo; principalmente, satisfacción laboral, engagement y compromiso con la tarea (Bakker, Rodríguez-Muñoz, \& Sanz Vergel, 2016; Mäkikangas, 2018; Petou et al., 2012; Rudolph et al., 2017; Tims, Bakker, Derks, \& van Rhenen, 2013; Zhang et al. 2018).

El desarrollo sostenido de la investigación empírica se ha visto estimulado por el diseño de instrumentos de medida válidos y confiables. En este sentido, si bien se han elaborado escalas especificas para grupos ocupacionales particulares, tales como obreros (Nielsen \& Abildgaard, 2012) y maestros (Leana, Appelbaum, \& Shevchuk, 2009), la publicación de la escala desarrollada por Tims et al. (2012) ha sido la principal impulsora de la investigación en el área. Esto se debe, posiblemente, a que constituye un instrumento genérico para medir el job crafting independientemente de la profesión y de la posición en el organigrama organizacional. 
Dicho instrumento se inscribe en los lineamientos teóricos del modelo DRL (Bakker \& Demerouti, 2017). La validación inicial de la Escala de Job Crafting fue realizada en Holanda, a partir de la información proporcionada por tres estudios complementarios ejecutados sobre trabajadores de diversos rubros $(n=1181)$. Análisis factoriales indicaron una solución de 21 ítems distribuidos entre cuatro factores denominados: (a) Aumento de los recursos estructurales, concerniente a aquellas acciones del empleado que tienen por objetivo desarrollar sus capacidades a través del aprendizaje y de la autonomía en los procesos de trabajo; (b) Aumento de los recursos sociales, relativa a la búsqueda y establecimiento de relaciones interpersonales positivas, basadas en la confianza, el apoyo y la cooperación; (c) Aumento de las demandas laborales desafiantes, relacionada a la asunción de nuevas tareas y a la iniciativa de participar en proyectos laborales retadores e interesantes, y (d) Disminución de las demandas laborales obstaculizantes, vinculada a las acciones orientadas a reducir o neutralizar aquellos aspectos del trabajo que suponen para el empleado un alto nivel de exigencias o costes psicológicos o físicos. Si bien estas dimensiones pueden considerarse de forma independiente, algunos autores (Akkermans \& Tims, 2017; Bell \& Njolli, 2016; Carvalho-Chinelato et al., 2015; Ingusci, Callea, Chirumbolo, \& Urbini, 2016; Rudolph et al., 2017; Tims et al., 2016), señalan que pueden agregarse en un puntaje general, sugiriendo asi que reflejarian un constructo latente de orden superior.

La escala desarrollada por Tims et al. (2012) ha alcanzado gran consenso entre la comunidad científica. No obstante, a pesar de su popularidad, persisten algunas contradicciones respecto a su estructura subyacente y al funcionamiento de algunos de sus ítems. En este sentido, mientras que algunos estudios (Bakker et al., 2018; Lichtenthaler \& Fischbach, 2016; Sora, Caballer, \& García-Buades, 2018) replicaron la estructura tetra-factorial informada en el estudio original de Tims et al. (2012), otras investigaciones arribaron a soluciones diversas. Así, por ejemplo, las validaciones del instrumento ejecutadas en Brasil (Carvalho-Chinelato et al., 2015) e Italia (Cenciotti et al., 2016) concluyeron que la dimensión Disminución de las demandas obstaculizantes no emergía como un factor idóneo. Del mismo modo, una adaptación turca (Rofcanin, Berber, Koch, \& Sevinc, 2015) señaló que un modelo de tres factores (en el que los recursos sociales y estructurales forman parte de la misma dimensión) era el que mejor se ajustaba a la matriz empírica de varianzas y covarianzas. Finalmente, un estudio japonés (Euguchi et al., 2016) encontró que un modelo de cinco factores (donde Disminución de las demandas obstaculizantes se dividía en dos factores denominados Demandas emocionales y Demandas cognitivas) emergía como una estructura más precisa que un modelo tetra-factorial.

Sumado a esto, según lo informan estudios instrumentales desarrollados en España (Bakker et al., 2018; Sora et al., 2018), Sudáfrica (Peral \& Geldenhuys, 2016), Turquía (Bacaksiz, Seren, \& Tuna, 2018), China (Zhang et al., 2018), Brasil (Carvalho-Chinelato et al., 2015) e Italia (Cenciotti et al., 2016), algunos ítems de la escala original no presentaron un funcionamiento adecuado. En este sentido, o bien presentaron cargas factoriales inferiores a lo recomendado $(\lambda<.40)$ por los especialistas (Lloret-Segura, Ferreres, Hernández, \& Tomás, 2017), o covarianzas entre los términos de error. Estas últimas debidas, probablemente, a la redundancia o superposición de contenido entre los ítems. Frente a estos resultados contradictorios, resulta 
legitimo que nuevas investigaciones proporcionen evidencia adicional sobre la validez interna de la escala. Por tanto, el objetivo de este estudio fue proporcionar evidencias de validez y confiabilidad de la Escala de Job Crafting desarrollada por Tims et al. (2012) en una muestra multi-ocupacional de trabajadores argentinos.

\section{MÉTODO}

\section{Diseño}

La presente investigación se inscribe en la categoria de estudios instrumentales, desde el momento en que se orienta al análisis psicométrico de un instrumento de medida (Ato, López, \& Benavente, 2013). Con tal propósito, se ejecutó un primer estudio orientado a traducir y adaptar el instrumento, así como a explorar la estructura subyacente a los ítems (Estudio 1); y un segundo estudio orientado a confirmar la estructura interna de la escala y a obtener evidencias de confiabilidad y validez (Estudio 2).

\section{ESTUDIO 1}

\section{Participantes}

A partir de la recomendación de contar con un mínimo de 200 observaciones para asegurar que la solución factorial fuera estable y generalizable (Lloret-Segura et al., 2017), se conformó una muestra por disponibilidad de 229 trabajadores de diversas organizaciones públicas y privadas de la ciudad de Rosario. El 56\% $(n=128)$ de la muestra fueron varones. El promedio de edad fue 33 años $(D E=6.24)$ y la antigüedad laboral fue de 7 años $(D E=3.81)$. El $71 \%(n=162)$ de la muestra se desempeñaba en el ámbito privado. El $72 \%(n=165)$ desarrollaba sus actividades en el rubro comercio y servicios (incluyendo en esta categoria telecomunicaciones, finanzas, hotelería, educación, salud y administración pública) y el $28 \%$ ( $n=$ 64) restante en el rubro industrial (principalmente textil y metalmecánica). E1 $57 \%(n=131)$ tenía estudios superiores (terciarios y/o universitarios). El $21 \%$ $(n=48)$ ocupaba posiciones de alta jerarquía en el organigrama organizacional (jefaturas, supervisiones, gerencias o direcciones).

\section{Instrumento}

Se utilizó la versión original traducida de la Escala de Job Crafting de Tims et al. (2012), conformada por 21 ítems orientados a evaluar cuatro dimensiones: (a) Aumento de los recursos estructurales (cinco items, ej.: "Trato de aprender cosas nuevas"); (b) Aumento de los recursos sociales (cinco items, ej.: "Solicito a mis compañeros consejos"); (c) Aumento de las demandas desafiantes (cinco ítems, ej.: "Cuando no hay mucho que hacer inicio nuevos proyectos"); y (d) Disminución de las demandas obstaculizantes (seis items, ej.: "Evito estar concentrado muchas horas"). Los items se presentaron en un formato tipo Likert de cinco puntos $(1=$ nunca; $5=$ siempre $)$. La confiabilidad informada por los autores fue satisfactoria, variando entre $\mathrm{a}=.75 \mathrm{y} \mathrm{a}=.82$ (Tims et al., 2012). 


\section{Procedimiento}

Se extendió la invitación a participar del estudio a diversas organizaciones pequeñas y medianas de la ciudad de Rosario. En algunos casos el contacto con las mismas se realizó por la intermediación de algún empleado que actuó como facilitador del acceso a la organización. En otros, se contactó directamente a las autoridades institucionales, coordinando una entrevista previa. Dado que se apeló a un muestreo por disponibilidad, y a que no se disponía de contactos con grandes organizaciones, estas últimas quedaron excluidas involuntariamente de la muestra final.

En todos los casos se explicitaron los objetivos y alcances de la investigación, así como el tratamiento confidencial y anónimo de la información provista por los empleados. Asimismo, se asumió el compromiso de devolución de los resultados, bajo el formato de un informe escrito o de presentación oral, según la elección de la organización. Se pautaron dias y horarios para efectuar la recolección de los datos. La misma se ejecutó en el seno de las organizaciones en los lugares fisicos que las mismas dispusieron a tal efecto. Los respectivos departamentos de recursos humanos, comunicación y/o desarrollo se encargaron de extender la invitación a participar del estudio a todos los empleados. La participación de estos fue voluntaria, anónima y confidencial. No se brindaron incentivos de ningún tipo. Quienes aceptaron colaborar brindaron su consentimiento, firmando el correspondiente protocolo. Previamente a responder, recibieron instrucciones precisas en forma oral con miras a completar la escala de modo individual. El tiempo aproximado que demandó completar el cuadernillo fue de 15 minutos. La administración de las técnicas estuvo a cargo de personal entrenado. Una vez concluida la administración, se habilitó un espacio para que los participantes pudieran opinar del instrumento, principalmente en lo referente a posibles ambigüedades de sentido, claridad de la redacción, tiempo requerido para completarla, etc. Se tomó nota de tales observaciones, las que fueron tenidas en cuenta para perfeccionar el instrumento, facilitando los ajustes semánticos y sintácticos necesarios. La ejecución del estudio se llevó a cabo en un todo de acuerdo con los lineamientos éticos establecidos por la Asociación Americana de Psicologia (American Psychological Association, 2017) y las recomendaciones del CONICET (Consejo Nacional de Investigaciones Científicas y Técnicas, 2006) para las investigaciones en las ciencias sociales y humanas.

\section{Estrategia de análisis}

Se analizó la validez de contenido, la equivalencia semántica y la equivalencia operacional entre el instrumento foráneo y su versión adaptada (Muñiz, Elosua, \& Hambleton, 2013). En este sentido, se efectuaron tareas de revisión bibliográfica y consultas con expertos para establecer el significado del constructo tanto en la cultura donde fue desarrollado el instrumento, como en la cultura objetivo. Como parte de este proceso, los reactivos de la escala también fueron sometidos a revisión crítica con el fin de establecer la equivalencia de ítems. En este sentido, tres especialistas (dos en psicología organizacional y uno en psicometria) recibieron un cuadernillo conteniendo definiciones conceptuales y operacionales del constructo, así como la totalidad de los ítems que integraban la escala. Se solicitó a los expertos que clasificaran cada ítem en la dimensión correspondiente. Sobre la base de tales 
evaluaciones, se efectuó un cálculo de consistencia a través del estadístico Kappa.

Aunque existe una versión del instrumento en lengua española (Bakker et al., 2018; Sora et al., 2018), dadas las particularidades léxicas y culturales de la población argentina, se decidió tomar como punto de partida la versión original publicada en inglés (Tims et al., 2012). La adaptación lingüística y el análisis de equivalencia semántica del instrumento se realizó siguiendo un procedimiento iterativo de traducción y re-traducción de acuerdo con el cual: (1) dos traductores profesionales tradujeron los items desde el inglés al idioma de la población objetivo (español-argentino); (2) otros dos profesionales bilingües retradujeron los ítems desde el español al inglés y (3) dos nuevos traductores científicos compararon a ciegas las dos formas del instrumento.

La equivalencia operacional (relativa a la posibilidad de mantener estables las características operativas durante la aplicación del instrumento) y la equivalencia métrica (referida a la estructura subyacente a los items), se determinaron a partir de los datos proporcionados por la muestra. En este sentido, se analizó la distribución de las variables mediante el cálculo de estadísticos descriptivos (medias y desviaciones estándar) y coeficientes de asimetría y curtosis univariada. Se determinó la capacidad de discriminación a través del cálculo de la correlación items-puntaje total (Tabachnick \& Fidell, 2013). Se ejecutó un análisis factorial exploratorio con el programa Factor (Lorenzo-Seva \& Ferrando, 2006), previa obtención de los índices de adecuación muestral. Por la naturaleza ordinal de los datos, se utilizó la matriz de correlaciones policóricas y el método de mínimos cuadrados no ponderados. Se llevó a cabo un análisis paralelo optimizado extrayendo aleatoriamente 5000 sub-matrices e implementando el análisis de rango mínimo y la rotación oblicua Promin, ya que existía la presunción de que los elementos de la escala estuvieran relacionados. El criterio para la selección de los items fue que pesaran .30 o más sobre el factor y que no saturaran sobre más de un factor al mismo tiempo (Lloret-Segura et al., 2017). La confiabilidad preliminar se estableció sobre la base del coeficiente alfa de Cronbach, tal como se hizo en el estudio original (Tims et al., 2012).

\section{RESULTADOS}

\section{Validez de contenido y equivalencia semántica}

El análisis de la literatura especializada (Carvalho-Chinelato et al., 2015; García-Cifuentes \& Sánchez Arroyo, 2015; Marcaccio \& Moreno, 2014; Nielsen, Antino, Sanz-Vergel, \& Rodríguez-Muñoz, 2017; Rotzinger, 2018) permitió concluir que el constructo job crafting es equivalente en ambas culturas. La revisión del contenido de cada ítem por parte de jueces expertos indicó que los mismos cubrian las cuatro dimensiones postuladas por la teoría (Demerouti, 2014; Demerouti \& Bakker, 2014; Tims \& Bakker, 2010; Tims et al., 2012). Los índices de concordancia inter-jueces calculados a partir de la clasificación de los ítems fueron altamente satisfactorios, oscilando entre $.81 \mathrm{y}$ $.90(p<.000)$. Respecto a la adaptación lingüística, ambos profesionales indicaron que se había mantenido inalterada la concordancia entre cada item original y el traducido al español, por lo que concluyeron que la versión argentina de la Escala de Job Crafting presentaba una adecuada equivalencia semántica. 


\section{Equivalencia operacional y métrica}

Los participantes señalaron que las instrucciones para realizar la tarea estaban claramente redactadas, que no tuvieron inconvenientes para comprender el contenido de los items y que la escala tipo Likert utilizada para responder no generaba dificultades. Los coeficientes de asimetría y curtosis univariada se encontraron comprendidos entre los valores de +/- 2, considerados adecuados por la literatura especializada (Tabachnick \& Fidell, 2013). Del mismo modo, las correlaciones ítem-total fueron todas positivas y superiores al valor mínimo esperado $(r=.30)$, según las recomendaciones de los expertos (Lorenzo-Seva \& Ferrando, 2006; Lloret-Segura et al., 2017).

La prueba de esfericidad de Bartlett fue significativa $\left(x^{2}{ }_{(136 ; 229)}=2356.13\right.$, $p<.001$ ) y el índice de adecuación muestral fue satisfactorio (Î́ndice KaiserMeyer-Olkin= .82). El análisis paralelo optimizado sugirió 4 factores con valores propios $(26.0,14.3,10.1$ y 8.2) sobre el percentil 95 de los valores propios obtenidos en las muestras aleatorias (12.1, 12.7, 8.8 y 6.2). Los 21 items presentaron saturaciones factoriales mayores o iguales a $.30, \mathrm{y}$ se distribuyeron de acuerdo con la estructura original: Aumento de recursos estructurales (Factor I, 5 items, a = .79); Aumento de recursos sociales (Factor II, 5 items, $\mathrm{a}=.84$ ); Aumento de desafios laborales (Factor III, 5 items, $\mathrm{a}=.77$ ) y Disminución de demandas laborales obstaculizantes (Factor IV, 6 items, a = .82). La varianza total del constructo explicada por los factores sugeridos fue del 60.61\% (Factor I = 24.19\%; Factor II = 17.85\%; Factor III = 10.35\%; Factor $\mathrm{IV}=8.22 \%$ ). En la tabla 1 se informan los estadísticos descriptivos, coeficientes de asimetría y curtosis índices de discriminación y coeficientes patrón.

\section{DISCUSIÓN}

El objetivo de este estudio fue traducir y adaptar la Escala de Job Crafting de Tims et al. (2012) para su empleo en Argentina, así como a explorar la estructura subyacente a los items en la muestra analizada. La revisión de literatura (Carvalho-Chinelato et al., 2015; García-Cifuentes \& Sánchez Arroyo, 2015; Marcaccio \& Moreno, 2014; Nielsen et al., 2017; Rotzinger, 2018) permite concluir que el constructo job crafting es equivalente, a pesar de haber sido desarrollado en un contexto sociocultural diferente del nacional. La revisión del contenido de los ítems por parte de jueces expertos concluyó que los mismos cubrian las cuatro dimensiones postuladas por la teoria. Asimismo, la adaptación lingüística realizada al español-argentino no conllevó alteraciones de semánticas respecto de la versión original en inglés.

El análisis paralelo optimizado, en tanto, indicó una estructura de cuatro factores, que se correspondía con la estructura original informada por Tims et al. (2012). De este modo, los 21 items se distribuyeron en cuatro dimensiones denominadas Aumento de recursos estructurales, Aumento de recursos sociales, Aumento de desafios laborales y Disminución de demandas laborales obstaculizantes, respectivamente. La varianza total del constructo explicada por los factores sugeridos fue del $60.61 \%$. Tales hallazgos armonizan con los resultados informados por otros estudios instrumentales, tales como las adaptaciones españolas (Bakker et al., 2018; Sora et al., 2018) y alemana (Lichtenthaler \& Fischbach, 2016). La confiabilidad preliminar fue satisfactoria para los cuatro factores identificados, ubicándose el coeficiente alfa de Cronbach en el rango de .74 - .84, en sintonía con los valores 
informados por otros autores (Bakker et al., 2018; Lichtenthaler \& Fischbach, 2016; Sora et al., 2018; Tims et al., 2012).

Tabla 1.

Coeficientes patrón, estadísticos descriptivos, coeficientes de asimetría y curtosis e índices de discriminación correspondientes a los ítems de la Escala de Job Crafting $(n=229)$.

\begin{tabular}{|c|c|c|c|c|c|c|c|c|c|}
\hline \multirow{2}{*}{ CONTENIDO DEL ÍTEM } & \multicolumn{4}{|c|}{ Coeficientes patrón } & \multicolumn{5}{|c|}{ Análisis descriptivos } \\
\hline & $\mathbf{I}$ & II & III & IV & $M$ & $D E$ & As & Cs & $r_{\text {i-total }}$ \\
\hline 1. Intento desarrollar todo mi potencial & .68 & .11 & .22 & .13 & 3.21 & 1.27 & 0.34 & -0.36 & .50 \\
\hline 2. Decido cómo realizar las tareas & .55 & .08 & .16 & -.07 & 3.15 & 1.09 & -0.08 & 0.15 & .43 \\
\hline $\begin{array}{l}\text { 3. Me esfuerzo por crecer } \\
\text { profesionalmente }\end{array}$ & .77 & -.13 & .19 & .09 & 3.50 & 1.12 & 0.12 & -0.24 & .51 \\
\hline $\begin{array}{l}\text { 4. Me aseguro de utilizar mis capacidades } \\
\text { al máximo }\end{array}$ & .72 & .09 & .24 & .11 & 3.48 & 0.96 & 0.07 & 0.21 & .50 \\
\hline 5. Trato de aprender cosas nuevas & .49 & .12 & .18 & -.10 & 3.37 & 0.90 & 0.23 & 0.38 & .42 \\
\hline $\begin{array}{l}\text { 6. Pido a mi supervisor que me enseñe lo } \\
\text { que necesito aprender }\end{array}$ & .21 & .71 & .27 & .17 & 3.31 & 1.19 & 0.11 & 0.12 & .49 \\
\hline $\begin{array}{l}\text { 7. Pido a mis compañeros feedback sobre } \\
\text { mi desempeño }\end{array}$ & .19 & .68 & .13 & .23 & 3.29 & 1.32 & 0.51 & 0.35 & .60 \\
\hline $\begin{array}{l}\text { 8. Converso con mi jefe si está satisfecho } \\
\text { con mi trabajo }\end{array}$ & .13 & .73 & .16 & .15 & 3.52 & 1.04 & -0.09 & 0.11 & .53 \\
\hline 9. Tomo como modelo a mi supervisor & .08 & .45 & .20 & .19 & 3.43 & 0.97 & 0.31 & 0.27 & .48 \\
\hline $\begin{array}{l}\text { 10. Pido a mis colegas consejos } \\
\text { profesionales }\end{array}$ & .12 & .70 & .18 & .07 & 3.75 & 1.22 & -0.10 & 0.19 & .54 \\
\hline $\begin{array}{l}\text { 11. Realizo tareas adicionales, aunque no } \\
\text { me paguen extra por ellas }\end{array}$ & .25 & .15 & .60 & -.10 & 3.29 & 0.91 & 0.53 & 0.36 & .50 \\
\hline $\begin{array}{l}\text { 12. Cuando hay un proyecto interesante } \\
\text { me ofrezco para participar }\end{array}$ & .20 & .25 & .65 & -.07 & 3.45 & 1.32 & -0.12 & 0.41 & .43 \\
\hline $\begin{array}{l}\text { 13. Cuando no tengo mucho que hacer en } \\
\text { mi trabajo empiezo tareas nuevas }\end{array}$ & .26 & .18 & .63 & -.09 & 3.88 & 1.21 & -0.35 & 0.06 & .51 \\
\hline $\begin{array}{l}\text { 14. Si hay técnicas y procedimientos } \\
\text { nuevos soy el primero en aprenderlos }\end{array}$ & .31 & .13 & .56 & -.05 & 3.70 & 1.25 & 0.13 & -0.25 & .53 \\
\hline $\begin{array}{l}\text { 15. Intento hacer que mi trabajo sea más } \\
\text { desafiante }\end{array}$ & .27 & .11 & .45 & -.18 & 3.31 & 0.92 & 0.21 & -0.26 & .44 \\
\hline $\begin{array}{l}\text { 16. Organizo mi trabajo para que sea } \\
\text { mentalmente lo menos estresante posible }\end{array}$ & .28 & .17 & -.04 & .63 & 3.23 & 1.21 & 0.32 & -0.21 & .60 \\
\hline $\begin{array}{l}\text { 17. Organizo mis tareas para evitar estar } \\
\text { concentrado muchas horas seguidas }\end{array}$ & .27 & .15 & .09 & .72 & 3.29 & 1.32 & 0.51 & 0.35 & .58 \\
\hline $\begin{array}{l}\text { 18. Trato de evitar el contacto con } \\
\text { personas cuyos problemas me afectan } \\
\text { 19. Intento que mi trabajo sea }\end{array}$ & -.16 & -.21 & -.17 & .69 & 3.28 & 1.26 & 0.29 & 0.30 & .57 \\
\hline $\begin{array}{l}\text { emocionalmente lo menos estresante } \\
\text { posible }\end{array}$ & .21 & .08 & .09 & .48 & 3.38 & 0.93 & 0.31 & -0.26 & .42 \\
\hline $\begin{array}{l}\text { 20. Trato de asegurarme no tener que } \\
\text { tomar decisiones difíciles en el trabajo* }\end{array}$ & .15 & .05 & .10 & .35 & 3.18 & 0.87 & 0.99 & -1.86 & .33 \\
\hline $\begin{array}{l}\text { 21. Minimizo el contacto con personas } \\
\text { cuyas expectativas no son realistas* }\end{array}$ & -.07 & -.19 & -.11 & .31 & 3.09 & 0.76 & -1.45 & 1.87 & .29 \\
\hline
\end{tabular}

Nota: Factor I: Aumento de recursos estructurales; Factor II: Aumento de recursos sociales; Factor III: Aumento de desafíos laborales; Factor IV: Disminución de demandas laborales obstaculizantes. As: Coeficiente de asimetría; Cs: Coeficiente de curtosis; $r$ i-total: Correlación ítem-total corregida. * = Ítems eliminados tras AFC. 


\section{ESTUDIO 2}

\section{Participantes}

Apelando a un muestreo no probabilístico por disponibilidad, se integró una muestra inicial de 454 participantes $(67.6 \%$ mujeres; $n=307 ; 32.4 \%$ varones; $n=147$ ) de diversas organizaciones públicas y privadas localizadas en el centro-sur de la provincia de Santa Fe. El promedio de edad fue de 34.5 años $(D E=12.94)$. La antigüedad laboral media fue de 8.6 años $(D E=10.21)$. E1 $84.5 \%(n=384)$ de la muestra tenia estudios superiores terciarios y/ o universitarios. E1 55\% $(n=250)$ trabajaba en el sector privado. La distribución por rubros de actividad fue la siguiente: $32.2 \%(n=146)$ industria; $30.5 \%(n=$ $138)$ comercio; $25.3 \%(n=115)$ salud y $12 \%(n=55)$ educación.

\section{Procedimiento}

Se extendió la invitación a participar del estudio a diversas organizaciones de la ciudad de Rosario, procurando que en la muestra final estuviesen representadas organizaciones de diverso tamaño y rubro de actividad. En algunos casos, el contacto con las mismas se realizó por la intermediación de algún empleado que actúo como facilitador del acceso a la organización. En otros, se contactó directamente a las autoridades institucionales o a los responsables de recursos humanos y desarrollo de las empresas. En todos los casos, se explicitaron los objetivos y alcances de la investigación, así como el tratamiento confidencial y anónimo de la información provista por los empleados. Asimismo, se asumió el compromiso de devolución de los resultados, bajo el formato de un informe escrito o de presentación oral según la elección de la organización.

Se pautaron dias y horarios para efectuar la recolección de los datos. La misma se ejecutó en el seno de las organizaciones en los lugares físicos que estas dispusieron a tal efecto. Los respectivos departamentos de recursos humanos, comunicación y/o desarrollo se encargaron de extender la invitación a participar del estudio a todos los empleados. La participación de estos fue voluntaria, anónima y confidencial. No se brindaron incentivos de ningún tipo. Quienes aceptaron colaborar brindaron su consentimiento firmando el correspondiente protocolo. Previamente a responder, recibieron instrucciones precisas en forma oral y escrita con miras a completar la escala de modo individual. El tiempo aproximado que demandó completar el cuadernillo fue de 30 minutos. La administración de las técnicas estuvo a cargo de personal entrenado. La ejecución del estudio se llevó a cabo en un todo de acuerdo con los lineamientos éticos establecidos por la Asociación Americana de Psicologia (American Psychological Association, 2017) y las recomendaciones del CONICET (Consejo Nacional de Investigaciones Científicas y Técnicas, 2006) para las investigaciones en las ciencias sociales y humanas.

\section{Instrumentos}

Job Crafting. Conceptualizado como una constelación de conductas automotivadas orientadas alinear el trabajo a las preferencias, competencias y aspiraciones del trabajador, mediante la gestión efectiva de las demandas y/o recursos laborales (Tims et al., 2013), fue medido mediante la versión traducida y adaptada en el Estudio 1 de la escala de Tims et al. (2012). Dicho instrumento se compone de 21 ítems presentados en formato tipo Likert de 
cinco puntos $(1=$ nunca; $5=$ siempre $)$, distribuidos en cuatro dimensiones: (a) Aumento de los recursos estructurales (cinco items, ej.: "Trato de aprender cosas nuevas"; $a=.79$ ); (b) Aumento de los recursos sociales (cinco ítems, ej.: "Solicito a mis compañeros consejos"; $a=.84$ ); (c) Aumento de las demandas desafiantes (cinco ítems, ej.: "Cuando no hay mucho que hacer inicio nuevos proyectos"; $a=.74) ; y(d)$ Disminución de las demandas obstaculizantes (seis items, ej.: "Evito estar concentrado muchas horas"; $a=.82$ ).

Personalidad Proactiva. De acuerdo con su definición conceptual, se trata de una tendencia relativamente estable a generar cambios en el ambiente en lugar de verse constreñido a actuar por presiones o requerimientos externos. Las personas con personalidades proactivas se destacan por su inclinación a identificar oportunidades de mejora, mostrar iniciativa, enfrentar los acontecimientos activamente, comprometerse más allá de lo estrictamente requerido y asumir con optimismo los desafios (Salessi \& Omar, 2017). Para su medición, se administró la versión breve de la escala homónima elaborada por Bateman y Crant (1993), en su versión adaptada y validada para la Argentina (Salessi \& Omar, en prensa). El instrumento está integrado por 10 items (ej.: "Siempre estoy buscando formas de hacer mejor las cosas"; $a=.80$ ) valorados sobre una escala Likert de cinco puntos $(1=$ totalmente en desacuerdo; 5 = totalmente de acuerdo).

Capital Psicológico. Conceptualmente, se define como el estado psicológico positivo, caracterizado por tener confianza (auto-eficacia) para asumir y llevar a cabo con éxito una tarea desafiante; hacer una atribución positiva (optimismo) sobre el resultado de la misma; perseverar en la búsqueda de los objetivos, redireccionando los esfuerzos hacia las metas en caso de ser necesario (esperanza), y a pesar de estar acosado por los problemas, fortalecerse en la adversidad (resiliencia) para alcanzar el éxito (Luthans \& Youssef-Morgan, 2017). Para su medición se utilizó la escala desarrollada por Omar, Salessi, y Urteaga (2014). Dicho instrumento está integrado por 16 items que evalúan los cuatro componentes del supra-factor: Esperanza (cuatro ítems, ej.: "Tengo fe en que mis problemas laborales van a mejorar"; $a=.80$ ); Optimismo (cuatro items, ej.: "Miro el lado positivo de cada proyecto laboral que emprendo"; $\mathrm{a}=.82$ ); Resiliencia (cuatro ítems, ej.: "Cuando tengo una dificultad en mi trabajo la supero con éxito"; $a=.86$ ), y Autoeficacia (cuatro ítems, ej.: "Si me esfuerzo puedo lograr los objetivos que me propongo"; $a=.88$ ). Los reactivos son presentados bajo un formato Likert de cinco puntos $(1=$ totalmente en desacuerdo; $5=$ totalmente de acuerdo).

Implicación en el Trabajo. Constituye un estado cognitivo de identificación psicológica con el trabajo, basado en la creencia en la capacidad de este para satisfacer las necesidades psicosociales y materiales más importantes del individuo (Salessi \& Omar, 2019). Para su medición se aplicó la versión argentina (Salessi \& Omar, 2018) del instrumento desarrollado por Kanungo (1982). El instrumento está integrado por nueve ítems (ej.: "Mi trabajo ocupa un lugar irremplazable en mi vida"; $a=.89$ ) valorados sobre una escala Likert de cinco puntos $(1=$ totalmente en desacuerdo; $5=$ totalmente de acuerdo).

\section{Estrategia de análisis}

El procesamiento y análisis de datos se realizó con los programas SPSS (versión 22.0), y EQS (versión 6.3). La estrategia adoptada para el análisis se detalla a continuación. 
Análisis exploratorios. Se examinaron los datos con miras a detectar la presencia de valores perdidos y puntuaciones extremas. El análisis de datos perdidos consistió, principalmente, en valorar su proporción y la posible presencia de sesgos en su distribución. Para ello, se aplicó la prueba de aleatoriedad de Little. Los casos atípicos se identificaron mediante el cálculo de puntuaciones $Z$ y de las distancias de Mahalanobis al cuadrado. Se analizó la distribución de las variables mediante el cálculo de estadísticos descriptivos (medias y desviaciones estándar), coeficientes de asimetría y curtosis, e índices de discriminación (a partir del cómputo de las correlaciones item-total corregidas) para cada uno de los ítems. Se computó el coeficiente de normalidad multivariado de Mardia normalizado (Bentler, 2006; Hair, Black, Babin, Anderson, \& Tatham, 2010; Tabachnick \& Fidell, 2013).

Evidencias de estructura interna. El modelo tetra-factorial sugerido por la teoria (Tims et al., 2012) y por el análisis factorial exploratorio desarrollado en el Estudio 1 fue contrastado mediante un análisis factorial confirmatorio. Para la estimación de los parámetros se empleó el método de máxima verosimilitud con la corrección robusta de Satorra-Bentler (Bentler, 2006), dada la naturaleza ordinal de los datos. Para evaluar la bondad de ajuste se emplearon los mismo indicadores y valores de referencia aplicados por Tims et al. (2012). En este sentido, se analizó que: (a) el índice $S B x^{2}$ sobre los grados de libertad $\left(S B x^{2} / g l\right)$ fuera inferior a 3; (b) el Índice de Ajuste Incremental (IFI, del inglés Incremental Fit Index), el Índice de Ajuste Comparativo (CFI, del inglés Comparative Fit Índex) y el Índice de Tucker-Lewis (TLI, del inglés Tucker-Lewis Índex) alcanzaran valores superiores a .90, y (c) el Error Cuadrático Medio de Aproximación (RMSEA, del inglés Root Mean Square Error of Approximation) fuera inferior a .08 (Hair et al., 2010).

Evidencias de confiabilidad. Las evidencias de confiabilidad se establecieron a partir del cómputo del coeficiente de confiabilidad compuesta (CR, Composite Reliability) y del coeficiente Omega de McDonald $(\omega)$. Valores de CR por encima de .70 se consideran evidencia de adecuada confiabilidad (Hair et al., 2010). El coeficiente $\omega$ es un estimador de consistencia interna basado en las cargas factoriales, que indica la proporción de varianza atribuida a la totalidad de la varianza común. Valores entre .70 y .90 se consideran aceptables (Ventura-León \& Caycho 2017).

Evidencias de validez. La validez convergente-discriminante se determinó mediante el cálculo de la varianza media extraída (AVE, del inglés Average Variance Extracted) y de su raíz cuadrada, respectivamente. El AVE permite estimar la varianza común entre los indicadores y su factor latente, considerando que valores superiores a .50 indican que más del $50 \%$ de la varianza del constructo es debida a sus indicadores. Por su parte, valores de la raíz cuadrada del AVE superiores a la correlación entre los factores latentes demuestran que cada constructo comparte más varianza con sus indicadores que con los demás (Cheung \& Chang, 2017; Martínez-García \& Martínez-Caro, 2009). La validez concurrente se analizó a partir del cálculo de las correlaciones entre los constructos estudiados, mediante el cómputo del coeficiente de rho Spearman. 


\section{RESULTADOS}

\section{Análisis exploratorios}

El porcentaje de datos perdidos en cada ítem no superó el 5\%. La prueba de Little indicó que el patrón de valores perdidos respondía completamente al azar (MCAR; $x^{2}=4153.07, p>.05$ ). Estos datos fueron reemplazados matemáticamente por valores calculados a partir del método EM (expectaciónmaximización). Las correlaciones ítem-total fueron todas positivas. Los valores de asimetría y curtosis univariada fueron inferiores a $+/-2$ (Tabachnick \& Fidell, 2013). No obstante, el coeficiente de curtosis multivariante normalizado se ubicó fuera del intervalo $+/-3\left(\mathrm{CK}_{\text {Mardia }}=11.09\right)$ recomendado por Bentler (2006) ameritando, entonces, el uso de estimadores robustos en la ejecución del AFC. El contraste de Mahalanobis no indicó la existencia de outliers multivariados $(p>.001$; Hair et al., 2010). No obstante, el cálculo de puntuaciones estándar permitió identificar cinco casos atípicos univariados (valores $Z>/$-3; Tabachnick \& Fidell, 2013). Los mismo fueron eliminados, quedando la matriz definitiva conformada, entonces, por 449 casos.

\section{Evidencias de estructura interna}

La tabla 2 presenta los indices de ajuste correspondientes al modelo original y a sus re-especificaciones

Tabla 2.

Índices de ajuste del modelo de medida original y los modelos de medida re-especificados para la Escala de Job Crafting $(n=449)$

\begin{tabular}{|c|c|c|c|c|c|}
\hline MODELO & $\mathrm{SBX}{ }^{2} / \mathrm{gl}$ & IFI & CFI & TLI & RMSEA \\
\hline Tetra factorial con 21 indicadores observables & 4.93 & .80 & .81 & .81 & $.07, \mathrm{IC} 95 \%[.06 ; .09]$ \\
\hline $\begin{array}{l}\text { Tetra factorial con } 19 \text { indicadores observables sin } \\
\text { covarianzas entre términos de error }\end{array}$ & 3.61 & .87 & .89 & .86 & $.06, \mathrm{IC} 95 \%[.05 ; .08]$ \\
\hline $\begin{array}{l}\text { Tetra factorial con } 19 \text { indicadores observables } \\
\text { con covarianzas entre términos de error }\end{array}$ & 2.85 & .92 & .93 & .91 & $.05, \mathrm{IC} 95 \%[.04 ; .06]$ \\
\hline
\end{tabular}

Tal como puede observarse en la tabla 2, los indices obtenidos para el modelo tetra-factorial (Tims et al., 2012) integrado por cuatro factores de primer orden denominados: "Aumento de recursos estructurales", "Aumento de recursos sociales", "Aumento de desafios laborales" y, "Disminución de demandas obstaculizantes"; 21 ítems como indicadores observables y sus respectivos errores de medida, indicaron un ajuste apropiado, pero no óptimo. Frente a este panorama, se optó por la estrategia de re-especificación del modelo (Brown, 2015; Hair et al., 2010) analizando la información provista tanto por las cargas factoriales estandarizadas, como por los residuos de la matriz de las predicciones de la covarianza y correlación. Se decidió retener aquellos ítems con una saturación factorial superior a .40 (Hair et al., 2014; Lloret-Segura et al., 2017). Del mismo modo, se permitió la correlación de residuos en aquellos pares de items pertenecientes al mismo factor, siempre y cuando hubiese motivos fundados para ello (Brown, 2015).

El examen de las cargas factoriales mostró que el ítem 20 ("Trato de asegurarme no tener que tomar decisiones dificiles en el trabajo") y el item 21 ("Organizo mi trabajo de modo de minimizar el contacto con personas cuyas 
expectativas no son realistas"), ambos pertenecientes al factor Disminución de las demandas obstaculizantes, presentaban saturaciones inferiores al minimo establecido ( $\lambda=.21$ y $\lambda=.25$, respectivamente). Frente a estos resultados, se optó por eliminar tales indicadores y volver a calcular el ajuste del modelo. Si bien se observó una mejoría en todos los indicadores, el grado de ajuste podría ser mayor tomando en cuenta que, según los índices de modificación de Lagrange, habia covarianzas de error significativas entre dos items pertenecientes al factor Incremento de los recursos sociales (item 7: "Pido a mis compañeros feedback sobre mi desempeño" e ítem 10: "Pido a mis compañeros consejos profesionales"; $r=.43, p<.001$ ); así como entre dos ítems correspondientes al factor "Disminución de las demandas obstaculizantes (item 16: "Organizo mi trabajo para que sea mentalmente lo menos estresante posible" e ítem 17 "Organizo mi trabajo para que sea emocionalmente lo menos estresante posible; $r=.40, p<.001$ ). Considerando que se trataba de contenidos redundantes y que, en tales circunstancias la literatura (Brown, 2015; Hair et al., 2010) avala la decisión de añadir nuevos parámetros al modelo, se agregaron tales covarianzas, recalculando el ajuste. El modelo reespecificado mostró un ajuste satisfactorio, por lo que se conservó como solución definitiva. Las cargas factoriales estandarizadas $(\lambda)$ para el factor Aumento de recursos estructurales estuvieron comprendidas entre .48 y .74; entre .58 y .75 para el factor Aumento de recursos sociales; entre .50 y .77 para el factor Aumento de desafios laborales y entre .71 y .80 para el factor Disminución de demandas obstaculizantes. La figura 1 ilustra gráficamente el modelo de medida final correspondiente a la Escala de Job Crafting (versión adaptada).

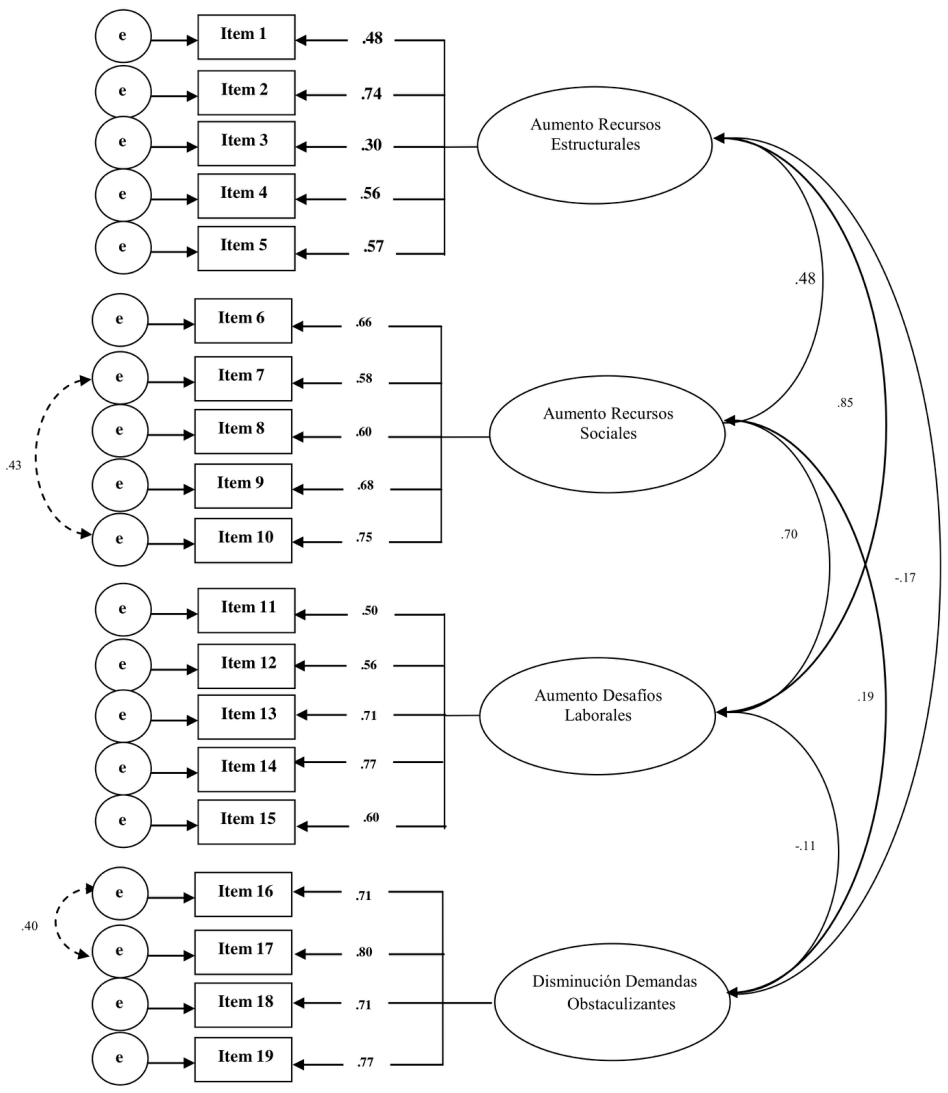

Figura 1. Modelo de medida correspondiente a la adaptación argentina de la Escala de Job Crafting de Tims et al. (2012). 


\section{Evidencias de confiabilidad, validez convergente-discriminante y concurrente}

La tabla 3 muestra los coeficientes de correlación entre las variables observables y entre las variables latentes, los estadísticos descriptivos ( $M \mathrm{y}$ $D E)$, los indices de confiabilidad (CR y $\omega$ ) y los valores correspondientes al indice AVE y a su raíz cuadrada.

Tabla 3.

Estadísticos descriptivos, coeficientes de confiabilidad, validez convergente-discriminante y correlaciones entre las variables observadas y latentes $(n=449)$.

\begin{tabular}{|c|c|c|c|c|c|c|c|c|c|c|c|c|}
\hline VARIABLES & $M$ & $D E$ & 1 & 2 & 3 & 4 & 5 & 6 & 7 & AVE & CR & $\omega$ \\
\hline $\begin{array}{l}\text { 1. Aumento recursos } \\
\text { estructurales }\end{array}$ & 3.34 & 1.11 & .84 & 10 & 1 & $16^{*}$ & ** & & * & .72 & .74 & .78 \\
\hline 2. Aumento recursos sociales & 3.45 & 1.26 & $.48^{* *}$ & .83 & $.60^{* *}$ & $.15^{*}$ & $.24^{* *}$ & $.39^{* *}$ & $.50^{* *}$ & .71 & .80 & .79 \\
\hline $\begin{array}{l}\text { 3. Aumento desafíos } \\
\text { laborales }\end{array}$ & 3.26 & 0.98 & $.85^{\star *}$ & .70 & 8 & 10* & $.44^{* *}$ & $.30^{\star *}$ & $.62^{* *}$ & .76 & .72 & .73 \\
\hline 4. Disminución demandas & 3.21 & 0.94 & $-.17^{*}$ & $.19^{*}$ & $-.11^{*}$ & .86 & $.14^{*}$ & $.09^{n s}$ & $.21^{*}$ & .74 & .80 & .80 \\
\hline 5. Personalidad proactiva & 3.28 & 1.30 & $.42^{\star \star}$ & $.23^{* *}$ & $.42^{* *}$ & $.11^{*}$ & .85 & $.21^{*}$ & $.49^{* *}$ & .73 & .81 & .79 \\
\hline vión on ol trahain & 3.85 & 1.24 & $.41^{* \star}$ & $.40^{* *}$ & $.33^{* *}$ & $.06^{n s}$ & $.18^{*}$ & .88 & $.42^{* *}$ & .78 & .79 & .81 \\
\hline 7. Capital psicológico & 3.42 & 1.21 & $.76^{\star *}$ & $.57^{\star \star}$ & $.65^{* *}$ & $.18^{\star *}$ & $.51^{* *}$ & $.44^{* *}$ & .87 & .76 & .82 & .80 \\
\hline
\end{tabular}

Nota: El significado de los números es: 1 (Aumento recursos estructurales); 2 (Aumento recursos sociales); 3 (Aumento desafíos laborales); 4 (Disminución demandas); 5 (Personalidad proactiva); 6 (Implicación en el trabajo); 7 (Capital psicológico). ${ }^{* *} p<.01 ;{ }^{*} p<.05 ; n s=$ no significativa. En negrita se informan las raíces cuadradas del AVE. Sobre la diagonal se presentan las correlaciones entre las variables observables. Bajo la diagonal se indican las correlaciones entre los factores latentes.

\section{DISCUSIÓN}

El objetivo de este segundo estudio fue confirmar la estructura factorial de la Escala de Job Crafting versión adaptada, obtenida en el Estudio 1, así como proporcionar evidencias de sus propiedades psicométricas. En lo que hace a las primeras, el AFC realizado corroboró la estructura tetra-factorial sugerida por el análisis paralelo. No obstante, cabe mencionar que aun cuando el modelo original de 21 ítems presentó un ajuste aceptable, el mismo no fue óptimo. Por este motivo se decidió buscar posibles re-especificaciones que se tradujeran en un mejor ajuste (Brown, 2015; Hair et al., 2010). Tales re-especificaciones resultaron en la eliminación de dos indicadores, los que no afectaron la configuración del modelo de medida.

En efecto, lejos de indicar una estructura nueva, los hallazgos que se desprenden de esta investigación aportan evidencia a favor de la organización tetra-partita a la que adscribe la teoría sobre job crafting (Bakker et al., 2018; Demerouti, 2014; Demerouti \& Bakker, 2014; Tims \& Bakker, 2010; Tims et al., 2012; Tims et al., 2013), desde el momento en que la reducción de los indicadores y la correlación entre los términos de error no implicaron ninguna reforma a nivel de los factores. Por el contrario, ambas re-especificaciones constituyen recursos legítimos, habida cuenta de que conservan la estructura general del modelo, pero únicamente con los indicadores más convenientes (Brown, 2015; Hair et al., 2010).

Los análisis realizados muestran que la eliminación de los dos reactivos tampoco perjudicó la confiabilidad de la escala. En este sentido, la versión adaptada del instrumento posee una adecuada consistencia interna comparable a la obtenida por Tims et al. (2012) y a la informada por otros estudios (Bakker et al., 2018; Cenciotti et al., 2016; Euguchi et al., 2016; Lichtenthaler \& Fischbach, 2016; Rofcanin et al., 2015; Sora et al., 2018). 
Del mismo modo, los valores obtenidos en el coeficiente AVE y en su raíz cuadrada permiten concluir que la varianza capturada por los cuatro factores identificados es mayor que la debida a los errores de medida, pudiéndose explicar adecuadamente a través de los indicadores elegidos. Tales hallazgos proporcionan evidencias favorables sobre la validez convergente y discriminante de la versión adaptada de la Escala de Job Crafting.

En lo que hace a las evidencias de validez concurrente, los resultados obtenidos muestran que el job crafting en todas sus dimensiones se asocia positivamente con la personalidad proactiva y con el capital psicológico. Hallazgos similares fueron comunicados por investigaciones precedentes. En este sentido, numerosos estudios han puesto de relieve las vinculaciones positivas entre la personalidad proactiva, el capital psicológico y los comportamientos proactivos individuales de rediseño del trabajo (Bakker et al., 2018; Berdicchia, et al., 2016; Carvalho-Chinelato, et al., 2015; Cenciotti et al., 2016; Rudolph et al., 2017; Zhang et al., 2018). En lo que respecta a la implicación, esta correlacionó positivamente con las dimensiones de Aumento de recursos estructurales, Aumento de recursos sociales y Aumento de los desafios laborales; mas no resultaron significativas las correlaciones con el factor Disminución de las demandas obstaculizantes. Hallazgos similares fueron informados por Bakker et al. (2018) y por otras investigaciones que examinaron las relaciones entre el job crafting y diversas formas de compromiso en el trabajo (Mäkikangas, 2018; Zhang et al. 2018).

\section{DISCUSIÓN GENERAL}

El estudio instrumental aquí presentado se orientó a adaptar y proporcionar evidencias de validez de la Escala de Job Crafting elaborada por Tims et al. (2012). El análisis paralelo optimizado indicó una estructura de cuatro factores, la que fue corroborada por el análisis factorial confirmatorio posterior. Dicho resultado armoniza con los hallazgos informados tanto por los autores del instrumento (Tims et al., 2012), como por las validaciones realizadas en España (Bakker et al., 2018; Sora et al., 2018) y Alemania (Lichtenthaler \& Fischbach, 2016).

Aun cuando la versión aquí presentada cuenta con algunas modificaciones, tales como la eliminación de dos ítems y la covarianza de dos pares de errores, el modelo de medida final no difiere sustancialmente del hallado por Tims et al. (2012). Lejos de indicar una estructura nueva, esta investigación aporta nueva evidencia a favor de la organización tetra-partita a la que adscribe la teoría sobre job crafting (Bakker et al., 2018; Demerouti, 2014; Tims \& Bakker, 2010; Tims et al., 2012; Tims et al., 2013).

Si bien en la validación inicial del instrumento (Tims et al., 2012) no se añadieron parámetros al modelo, las validaciones ejecutadas en otros países (Bacaksiz, et al., 2018; Bakker et al., 2018; Cenciotti et al., 2016; Euguchi et al., 2016; Lichtenthaler \& Fischbach, 2016; Peral \& Geldenhuys, 2016; Rofcanin et al., 2015) también han concluido que re-especificaciones como las aquí efectuadas son necesarias para obtener un ajuste óptimo entre el modelo teórico y la matriz empírica. No obstante, es sabido que añadir o suprimir parámetros solo es legitimo si existen razones fundadas para hacerlo. En este caso, se observa que las correlaciones entre los términos de error que fueron añadidas responden a los mismos pares de items que en otras adaptaciones (Bacaksiz, et al., 2018; Bakker et al., 2018; Euguchi et al., 2016; Peral \& 
Geldenhuys, 2016). Por lo que es posible concluir que tales covarianzas deben considerarse sistemáticas antes que errores aleatorios, pudiendo deberse a características especificas de los items. Por ejemplo, un alto grado de redundancia de contenido o superposición, tal como se observó en este caso. En lo que respecta a la supresión de indicadores observables, el mismo procedimiento ha sido aplicado en otros estudios sobre esta herramienta (Bacaksiz, et al., 2018; Lichtenthaler \& Fischbach; Peral \& Geldenhuys, 2016), constituyendo una práctica habitual en la investigación empírica instrumental con miras a incrementar el ajuste del modelo a los datos. En este caso, los items removidos corresponden a los mismos indicadores suprimidos por Sora et al. (2018) en el factor Disminución de las demandas obstaculizantes, en el marco de su estudio de validación de una versión breve de la Escala de Job Crafting de Tims et al. (2012).

Los valores obtenidos en el coeficiente AVE y su raíz cuadrada proporcionan evidencia de adecuada validez convergente-discriminante entre las cuatro facetas del constructo, así como entre estas y las restantes variables analizadas en este estudio. Al respecto, cabe destacar que en lo que hace a las inter-correlaciones entre las diversas facetas del job crafting, tres de sus dimensiones se asocian positivamente, mientras que la dimensión Disminución de demandas obstaculizantes presentó relaciones negativas con los factores Aumento de los recursos estructurales y Aumento de los desafios laborales, respectivamente. Lejos de ser un hallazgo inusual, dicho patrón ya ha sido informado en otros estudios (Bakker et al., 2018; Eguchi et al., 2016; Lichtenthaler \& Fischbach, 2016; Petrou et al., 2012; Sora et al., 2018) e, incluso, discutido por los propios autores de la escala (Tims et al., 2012).

$\mathrm{Si}$ bien sería esperable que las cuatro facetas se vinculasen positivamente entre sí (desde el momento en que todas constituyen comportamientos proactivos orientados a introducir cambios en el ambiente laboral), Tims et al. (2012) concluyeron que la evidencia empírica parece indicar que la disminución de demandas obstaculizantes representa un factor independiente de los otros tres. En este sentido, la ausencia de demandas obstaculizantes en el ambiente laboral dejaría más tiempo y energía disponible para crear trabajos más interesantes y desarrollar recursos personales. $\mathrm{O}$ bien, el incremento de recursos y desafios propiciaria un ambiente de trabajo placentero, caracterizado por la percepción de pocas demandas obstaculizantes que reducir. En cualquier caso, estos hallazgos podrian estar señalando que los mecanismos subyacentes que motivan a los empleados a aumentar sus recursos y metas retadoras pueden ser diferentes de los procesos que motivan a los empleados a disminuir los obstáculos que impiden un funcionamiento óptimo (Bakker et al., 2018; Demerouti, 2014; Tims et al., 2012).

Si bien los resultados obtenidos son auspiciosos, vale destacar en línea con el debate contemporáneo que la validez no es una cuestión de todo o nada y dista de ser una propiedad intrínseca de los instrumentos (Messick, 1980; Montero Rojas, 2013). Por el contrario, la validez se define de acuerdo con el propósito de la medición, la población a la que va dirigida y el contexto específico de aplicación. Así, un instrumento puede exhibir un grado aceptable de validez para un propósito específico y para una población particular, pero no para otros. En otras palabras, no se puede decir de manera contundente que un instrumento es válido, sino que exhibe un grado aceptable de validez para ciertos usos específicos y con ciertas poblaciones. Teniendo en cuenta 
tales advertencias, es preciso concluir que los hallazgos del presente estudio deben ser interpretados con cautela y dentro de los limites de la investigación ejecutada.

\section{Limitaciones, fortalezas y lineamientos para la investigación futura}

Entre las principales limitaciones de la investigación realizada hay que citar la representatividad de la muestra. Esta, al haber sido seleccionada en forma no probabilística, impide extrapolar los resultados obtenidos más allá de la muestra analizada. Sumado a eso, el haber aplicado un muestro por disponibilidad conllevó la no inclusión de organizaciones grandes, quedando conformada la muestra bajo estudio sólo por trabajadores de organizaciones PYMES.

Una segunda limitación podría estar vinculada con la validez externa del instrumento aquí presentado. Como no se ha verificado la estabilidad a lo largo del tiempo, sería recomendable que próximos estudios exploren la confiabilidad test-retest de esta versión. Cabe recordar que el job crafting, lejos de ser una conducta aislada o un evento en el tiempo, se configura como un proceso sostenido (Demerouti \& Bakker, 2014; Wrzesniewski \& Dutton, 2001). En efecto, investigaciones longitudinales (Nielsena \& Abildgaard, 2012; Nielsen et al., 2017; Tims et al., 2015) han mostrado adecuados coeficientes de estabilidad entre las diversas mediciones del constructo a lo largo de un lapso de 12 meses.

Del mismo modo, no haber obtenido otras evidencias de validez, tales como, por ejemplo, las de su capacidad predictiva, podría constituir una tercera limitación. Frente a este panorama, se alienta a que otras investigaciones examinen si las puntuaciones en esta escala constituyen predictores válidos y precisos de otras actitudes y comportamientos relevantes para las organizaciones y sus miembros. En este sentido, por ejemplo, sería interesante explorar si el job crafting resulta un antecedente significativo de los niveles de compromiso con la organización (más allá de la implicación con el trabajo que aquí se ha demostrado), así como de otros comportamientos que excedan los prescriptos por su rol (por ejemplo, conductas de ciudadanía organizacional, comportamientos innovadores, comportamientos contraproducentes, etc.). La investigación en esta área es aún muy exigua, por lo que invertir esfuerzos en esta dirección tendrá importantes implicaciones teóricas y prácticas.

Por último, cabe remarcar que, como se trata de un instrumento de autoinforme, las respuestas podrían verse contaminadas por la deseabilidad social. Por lo tanto, sería importante en futuros estudios obtener información de otras fuentes (tales como compañeros y supervisores), y utilizar mecanismos de validez interna (por ejemplo, escalas de deseabilidad social).

A pesar de estas limitaciones, el trabajo realizado constituye la primera tentativa de validar la Escala de Job Crafting de Tims et al. (2012) en Argentina, por lo que representa una contribución genuina a la literatura organizacional. Si bien retoma la linea de investigación inaugurada por Marcaccio y Moreno (2014), se focaliza en una escala diferente a la estudiada por tales autores, quienes adaptaron la escala de Nielsen y Abildgaard (2012). Mientras que dicho instrumento representa una herramienta idónea para evaluar los comportamientos individuales de rediseño entre los denominados trabajadores de cuello azul (obreros, operarios y personal con menor calificación), la escala aquí presentada constituye un instrumento para medir 
el job crafting independientemente de la ocupación o posición del empleado en la estructura organizacional. Sin embargo, dado su carácter general precisamente, la selección de demandas, recursos y desafios considerados resulta amplia y general. Vale decir que, si bien la escala contempla las demandas laborales más frecuentes y los recursos laborales más influyentes de acuerdo con la literatura (Bakker \& Demerouti, 2017; Demerouti, 2014; Demerouti \& Bakker, 2014; Tims \& Bakker, 2010; Tims et al., 2013), podría no ser una herramienta idónea si se pretende explorar exigencias, recursos y retos específicos de una profesión o puesto particular.

Frente a este panorama, se abre entonces la necesidad de llevar a cabo nuevos estudios instrumentales, orientados al desarrollo y validación de instrumentos más específicos para la evaluación del job crafting. Del mismo modo, considerando que cierta evidencia (Berg, Wrzesniewski, \& Dutton, 2010; Erez, 2010) indica que este tipo de conductas pueden variar en función del grado de calificación del trabajador y de los valores culturales, sería importante que futuras investigaciones profundicen estos hallazgos examinando, por ejemplo, la invarianza factorial de esta escala a la luz de tales aspectos. Asimismo, considerando que los hallazgos respecto a las intercorrelaciones entre las subescalas de job crafting sugieren cierta independencia del factor Disminución de las demandas obstaculizantes, sería necesario que próximos estudios profundicen esta cuestión. En este sentido, por ejemplo, sería conveniente indagar los posibles mecanismos mediadores que subyacen a las relaciones entre las variables antecedentes y las diversas manifestaciones del job crafting, operacionalizadas como aumento de recursos personales y/o sociales, incremento de retos laborales y disminución de presiones obstaculizantes. Cabe recordar que, a la luz de las correlaciones encontradas entre los factores, y de acuerdo con las advertencias de los especialistas (Bakker et al., 2018; Demerouti, 2014; Demerouti \& Bakker, 2014; Tims et al., 2012), es posible que los mecanismos subyacentes que motivan a los empleados a aumentar sus recursos y a proponerse metas retadoras sean diferentes de los procesos que motivan a los empleados a disminuir los obstáculos que impiden un funcionamiento óptimo.

\section{CONCLUSIÓN}

Desde su introducción en el ámbito académico (Wrzesniewski \& Dutton, 2001), el job crafting ha sido sindicado como una estrategia para incrementar la motivación de los trabajadores, fidelizar empleados y retener talentos. A través de la introducción de pequeños cambios en las demandas y recursos, no solo es posible lograr modificaciones en el diseño y el ambiente laboral, sino también en el significado del trabajo, en el compromiso del empleado y en sus satisfacción y bienestar general (Akkermans \& Tims, M., 2017; Bakker et al., 2016; Tims et. 2013). Por todo esto, el job crafting se revela como un modo simple y eficaz a través del cual las organizaciones pueden motivar y retener a sus empleados, especialmente cuando otros recursos (incrementos salariales, premios, promociones laborales, etc.) se encuentran limitados.

La escala aquí presentada se erige como una herramienta útil para la evaluación del job crafting en población hispanoparlante. Al tratarse de un instrumento con probadas propiedades psicométricas, se espera que pueda capitalizarse en futuros estudios orientados a examinar la presencia de este fenómeno en las organizaciones latinoamericanas. Si bien su principal utilidad se observa en el contexto de la investigación empírica, la elaboración de los 
correspondientes baremos facilitará una interpretación más rigurosa de la información proporcionada por la escala (Berg et al., 2010; Erez, 2010). Dicha información, a su vez, podrá contribuir al desarrollo de investigaciones aplicadas, orientadas al diseño de estrategias tendientes a promover la autonomia, el sentido control y la proactividad de los empleados frente a su trabajo.

\section{REFERENCIAS}

Akkermans J. \& Tims, M. (2017). Crafting your career: How career competencies relate to career success via job crafting. Applied Psychology: An International Review, 66(1), 168-195. https://doi.org/10.1111/apps.12082

American Psychological Association (2017). Ethical principles of psychologists and code of conduct [Electronic version]. Recuperado de http://www.apa.org/ethics/code

Ato, M., López, J., \& Benavente, A. (2013). Un sistema de clasificación de los diseños de investigación en psicologia. Anales de Psicología, 29(3), 1038-1059. https://doi.org/10.6018/analesps.29.3.178511

Bacaksiz, F., Seren, A., \& Tuna, R. (2018). Adaptation and validation of the job crafting behaviors of nurses scale in Turkish. International Journal of Caring Sciences, 11(1), 512-522.

Bakker, A. \& Demerouti, E. (2017). Job demands-resources theory: Taking stock and looking forward. Journal of Occupational Health Psychology, 22(3), 273-285. https://doi.org/10.1037/ocp0000056

Bakker, A., Ficapal-Cusí, P., Torrent-Sellens, J., Boada-Grau, J., \& Hontangas-Beltrán, P. (2018). The Spanish version of the Job Crafting Scale. Psicothema, 30(1), 136-1421.

Bakker, A., Rodríguez-Muñoz, A., \& Vergel, A. I. S. (2016). Modelling job crafting behaviors: Implications for work engagement. Human Relations, 69, 169-189. https://doi.org/10.1177/0018726715581690

Bateman, T. S. \& Crant, J. M. (1993). The proactive component of organizational-behaviour: A measure and $\begin{array}{lllll}\text { correlates. Journal of } & \text { Organizational }\end{array}$ https://doi.org/10.1002/job.4030140202

Bell, C. \& Njoli, N. (2016). The role of big five factors on predicting job crafting propensities amongst administrative employees in a South African tertiary institution. Journal of Human Resource Management, 14, 1-11. https://doi.org/10.4102/sajhrm.v14i1.702

Bentler, P. (2006). EQS 6 Structural Equations Program Manual. Los Angeles, CA: Multivariate Software Inc.

Berdicchia, D., Nicolli, F., \& Masino, G. (2016). Job enlargement, job crafting and the moderating role of self-competence. Journal of Managerial Psychology, 31, 318-330. https://doi.org/10.1108/JMP-012014-0019

Berg, J. M., Dutton, J. E., \& Wrzesniewski, A. (2013). Job crafting and meaningful work. En B. J. Dik, Z. S. Byrne, \& M. F. Steger (Eds.), Purpose and meaning in the workplace (pp. 81-104). Washington, DC: American Psychological Association. https://doi.org/10.1037/14183-005

Berg, J. M., Wrzesniewski, A., \& Dutton, J. E. (2010). Perceiving and responding to challenges in job crafting at different ranks: When proactivity requires adaptivity. Journal of Organizational Behavior, 31(2), 158-186. https://doi.org/10.1002/job.645

Brown, T. A. (2015). Confirmatory factor analysis for applied research. New York, NY: Guilford Publications.

Carvalho-Chinelato, R., Ferreira, M. C., \& Valentini, F. (2015). Evidence of validity of the Job Crafting Behaviors Scale. Paidéia, 25(62), 325-332. https://doi.org/10.1590/1982-43272562201506

Cenciotti, R., Borgogni, L., Callea, A., Colombo, L., Giovanni Cortese, C., Ingusci, E. ... Zito, M. (2016). The Italian version of the Job Crafting Scale. Applied Psychology Bulletin, 277(64), 28-36.

Cheung, G. \& Chang, W. (2017). Current approaches for assessing convergent and discriminant validity with SEM: Issues and solutions. Academy of Management Annual Meeting Proceedings, 1-1. https://doi.org/10.5465/AMBPP.2017.12706abstract

Consejo Nacional de Investigaciones Científicas y Técnicas (2006). Resolución D No. 2857: Lineamientos para el comportamiento ético en las Ciencias Sociales y Humanidades. Buenos Aires: CONICET.

Demerouti, E. (2014). Design your own job through job crafting. European Psychologist, 19(4), $237-247$. https://doi.org/10.1027/1016-9040/a000188

Demerouti, E. \& Bakker, A. (2014). Job crafting. En C. W. Peeters, J. Jonge \& T. Taris (Eds.), An Introduction to Contemporary Work Psychology (pp. 415-432). Chichester: Wiley-Blackwell.

Eguchi, H., Shimazu, A., Bakker, A., Tims, M., Kamiyama, K., Hara, J. ... Kawakami, N. (2016). Validation of the Japanese version of the Job Crafting Scale. Journal of Occupational Health, 58(3), 231-240. https://doi.org/10.1539/joh.15-0173-OA

Erez, M. (2010). Culture and job design. Journal of Organizational Behavior, 31(3), 389-400. https://doi.org/10.1002/job.651

García-Cifuentes, R. \& Sánchez Arroyo, M. (2015). Job Crafting en Chile. Un estudio exploratorio. Tesis de grado. Facultad de Ciencias Sociales. Universidad de Chile [Versión electrónica]. Recuperado de http://repositorio.uchile.cl/handle/2250/136440

Hair, J. E., Black, W. C., Babin, B. J., Anderson, R. E., \& Tatham, R. L. (2010). Multivariate data analysis (6th Ed.). Upper Saddle River, NJ: Pearson-Prentice Hall. https://doi.org/10.1016/j.jmva.2009.12.014

Ingusci, E., Callea, A., Chirumbolo, A., \& Urbini, F. (2016). Job crafting and job satisfaction in a sample of Italian teachers: The mediating role of perceived organizational support. Electronic Journal of Applied Statistical Analysis, 9, 675-687.

Kanungo, R. N. (1982). Measurement of job and work involvement. Journal of Applied Psychology, 67, 341- 
349. https://doi.org/10.1037/0021-9010.67.3.341

Leana, C., Appelbaum, E., \& Shevchuk, I. (2009). Work process and quality of care in early childhood education: The role of job crafting. Academy of Management Journal, 52(6), 1169-1192. https://doi.org/10.5465/amj.2009.47084651

Lichtenthaler, P., \& Fischbach, A. (2016). The conceptualization and measurement of job crafting: Validation of a German version of the Job Crafting Scale. Zeitschrift für Arbeits-und Organisationspsychologie 60(4), 173-186. https://doi.org/10.1026/0932-4089/a000219

Lloret-Segura., S., Ferreres, A., Hernández, A., \& Tomás, I. (2017). El análisis factorial exploratorio de los ítems: una guía práctica, revisada y actualizada. Anales de Psicología, 30(3), 1151-1169. https://doi.org/10.6018/analesps.30.3.199361

Lorenzo-Seva, U. \& Ferrando, P. (2006). FACTOR: A computer program to fit the exploratory factor analysis model. Behavior Research Methods, 38(1), 88-91. https://doi.org/10.3758/BF03192753

Luthans, F. \& Youssef-Morgan, C. (2017). Psychological capital: An evidence-based positive approach. Annual Review of Organizational Psychology and Organizational Behaviour, 4, 339-366. https://doi.org/10.1146/annurev-orgpsych-032516-113324

Mäkikangas, A. (2018). Job crafting profiles and work engagement: A person-centred approach. Journal of Vocational Behaviour, 106, 101-111. https://doi.org/10.1016/j.jvb.2018.01.001

Marcaccio, A. \& Moreno, J. (noviembre, 2014). Estudio preliminar de una medida de Job Crafting. Trabajo presentado en el VI Congreso Internacional de Investigación y Práctica Profesional en Psicología, XXI Jornadas de Investigación Décimo Encuentro de Investigadores en Psicología del MERCOSUR, Facultad de Psicologia-Universidad de Buenos Aires. Recuperado de https://www.aacademica.org/000-035/335.pdf

Martinez-García, J. \& Martínez-Caro, L. (2009). La validez discriminante como criterio de evaluación de escalas: ¿teoria o estadística? Universitas Psychologica, 8(1), 27-36.

Messick, S. (1980). Test validity and the ethics of assessment. American Psychologist, 35, 1012-1022. https://doi.org/10.1037/0003-066X.35.11.1012

Montero Rojas, E. (2013). Referentes conceptuales y metodológicos sobre la noción moderna de validez de instrumentos de medición: implicaciones para el caso de personas con necesidades educativas especiales. Actualidades en Psicología, 27(114), 113-128. https://doi.org/10.15517/ap.v27i114.7900

Muñiz, J., Elosua, P., \& Hambleton, R. (2013). Directrices para la traducción y adaptación de los tests: segunda edición. Psicothema, 25, 151-157.

Nielsen, K. \& Abildgaard, J. S. (2012). The development and validation of a job crafting measure for use with blue-collar workers. Work \& Stress, 26, 365-384. https://doi.org/10.1080/02678373.2012.733543

Nielsen, K., Antino, M., Sanz-Vergel, A., \& Rodríguez-Muñoz, A. (2017) Validating the Job Crafting Questionnaire (JCRQ): A multi-method and multi-sample study, Work \& Stress, 31(1), 82-99. https://doi.org/10.1080/02678373.2017.1293752

Omar, A., Salessi, S. \& Urteaga, F. (2014). Diseño y validación de una nueva escala para medir capital psicológico. Liberabit, 20(2), 315-323.

Peral, S. \& Geldenhuys, M. (2016). The effects of job crafting on subjective well-being amongst South African high school teachers. Journal of Industrial Psychology, 42(1), a1378, 1-13. https://doi.org/10.4102/ sajip.v42i1.1378

Petrou, P., Demerouti, E., \& Schaufeli, W. (2015). Job crafting in changing organizations: Antecedents, implications for exhaustion and performance. Journal of Occupational Health Psychology, 20(4), 470480. https://doi.org/10.1037/a0039003

Petrou, P., Demerouti, E., Peeters, M., Schaufeli, W. B., \& Hetland, J. (2012). Crafting a job on daily basis: Contextual antecedents and the link to work engagement. Journal of Organizational Behavior, 33, 1120-1141. https://doi.org/10.1002/job.1783

Rofcanin, Y., Berber, A., Koch, S., \& Sevinc, L. (2015). Job crafting and I-deals: A study testing the nomological network of proactive behaviors. The International Journal of Human Resource Management, 27(22), 2695-2726. https://doi.org/10.1080/09585192.2015.1091370

Rotzinger, J. (2018). A cross-cultural comparison on the mediating influence of job crafting between calling and creativity on satisfaction [Electronic Version]. Recuperado de http: arno.uvt.nl/show.cgi?fid=146185

Rudolph, C. W., Katz, I. M., Lavigne, K. N., \& Zacher, H. (2017). Job crafting: A meta-analysis of relationships with individual differences, job characteristics, and work outcomes. Journal of Vocational Behavior, 102, 112-138. https://doi.org/10.1016/j.jvb.2017.05.008

Salessi, S. \& Omar, A. (2017). Comportamientos proactivos en el trabajo: una puesta al día. Revista Argentina de Ciencias del Comportamiento, 9(3), 82-103

Salessi, S. \& Omar, A. (2018). Cuestionario de implicación en el trabajo. Propiedades psicométricas de su adaptación en Argentina. Revista Mexicana de Psicología, 35(2), 96-114.

Salessi, S. \& Omar, A. (2019). Jon Involvement in current research. Update and state of the art. Papeles del Psicólogo, 4O(1), 46-56. https://doi.org/10.23923/pap.psicol2019.2882

Salessi, S. \& Omar, A. (en prensa). Propiedades Psicométricas de la Versión Argentina de la Escala de Personalidad Proactiva. Psico USF.

Sora, B., Caballer, A., \& Garcia-Buades, E. (2018). Validation of a short form of Job Crafting Scale in a Spanish sample. The Spanish Journal of Psychology, 21, (e51), 1-13. https://doi.org/10.1017/sjp.2018.52

Tabachnick, B. \& Fidell, L. S (2013). Using Multivariate Statistics (6th Ed.). Boston, MA: Pearson.

Tims, M. \& Bakker, A. (2010). Job crafting: Towards a new model of individual job redesign. South African Journal of Industrial Psychology, 36, 1-9. https://doi.org/10.4102/sajip.v36i2.841

Tims, M., Bakker, A., \& Derks, D. (2012). Development and validation of the Job Crafting Scale. Journal of Vocational Behavior 80,173-186. https://doi.org/10.1016/j.jvb.2011.05.009 
Tims, M., Bakker, A., \& Derks, D. (2013). The impact of job crafting on job demands, job resources, and well-being. Journal of Occupational Health Psychology, 18(2), 230-240. https://doi.org/10.1037/a0032141

Tims, M., Bakker, A., \& Derks, D. (2014). Daily job crafting and the self-efficacy- performance relationship. Journal of Managerial Psychology, 29(5), 490-507. https://doi.org/10.1108/JMP-05-2012-0148

Tims, M., Bakker, A., \& Derks, D. (2015). Job crafting and job performance: A longitudinal study. European Journal of Work and Organizational Psychology, 24(6), 914-928. https://doi.org/10.1080/1359432X.2014.969245

Tims, M., Bakker, A., Derks, D., \& van Rhenen, W. (2013). Job crafting at the team and individual level: Implications for work engagement and performance. Group and Organization Management, 38(4), 427454. https://doi.org/10.1177/1059601113492421

Tims, M., Derks, D., \& Bakker, A. (2016). Job crafting and its relationships with person-job fit and meaningfulness: A three-wave study. Journal of Vocational Behavior, 92, 44-53. https://doi.org/10.1016/j.jvb.2015.11.007

van Dam, K., Nikolova, I., \& van Ruysseveldt, J. (2013). The importance of LMX and situational goal orientation as predictors of job crafting. Gedrag \& Organisatie, 26(1), 66-84.

Ventura-León, J. \& Caycho T. (2017). El coeficiente Omega: un método alternativo para la estimación de la confiabilidad. Revista Latinoamericana de Ciencias Sociales, Niñez y Juventud, 15(1), 625-627.

Walk, M. \& Handy, F. (2018). Job crafting as reaction to organizational change. The Journal of Applied Behavioral Science, 54(3), 349-370. https://doi.org/10.1177/0021886318777227

Wrzesniewski, A. \& Dutton, J. E. (2001). Crafting a job: Revisioning employees as active crafters of their work. Academy of Management Review, 26, 179- 201. https://doi.org/10.2307/259118

Zhang, L., Lu, H., \& Li, F. (2018). Proactive personality and mental health: The role of job crafting. Psychological Journal, 7(3), 154-155. https://doi.org/10.1002/pchj.214

Recibido 28-04-2019 | Aceptado 20-09-2019

Este trabajo se encuentra bajo una Licencia Creative Commons Atribución 4.0 Internacional que permite a terceros utilizar lo publicado siempre que se dé el crédito pertinente a los autores y a Psicodebate 